Sergio Trujillo García* 


\section{Algunas mostraciones de una tensión genealógica en las historias de las psicologías en Colombia}

Cómo citar este artículo: Trujillo García, S. (2018). Algunas mostraciones de una tensión genealógica en las historias de las psicologías en Colombia. Tesis Psicológica, 13(1), 104-114. https://doi.org/10.37511/tesis.v13n1a7
Recibido: 16 agosto 2017

Revisado: 24 agosto 2017

Aprobado: 21 junio 2018

\begin{abstract}
This article, about the history of psychology in Colombia, seeks to highlight different historical manifestations of the same founding tension, following a genealogical method, in the manner of Foucault and Nietzsche, which does not respect a linear chronological order, but assumes the spiral of history, in which we can find repetitions of the same categories, as Hegel enunciated and we have sustained in previous writings. Indeed, from a phrase published in the newspaper El Espectador in approximately 1978, the article returns to the conquest of South America where the same tension emerges with another face, that of the slave traders, then jumps to the proposals theoretical and empirical studies of a nineteenth-century author in the United States, considered the founder of a psychological school, to then consider the original proposals of psychometrics, which are brought from Europe, from the Geneva school to our country by Mercedes Rodrigo Bellido, who is considered founder of the first psychology faculty in the country. The article then reviews the purposes and intentions that Mercedes Rodrigo could have had, and contrast them with the eugenic ideas of some thinkers of that time and of today.
\end{abstract}

Keywords: history, psychology, genealogy, tension, emergency.

\section{RESUMEN}

Este artículo, acerca de la historia de la psicología en Colombia, busca resaltar diferentes mostraciones históricas de una misma tensión fundante, siguiendo un método genealógico, a la usanza de Foucault y de Nietzsche, que no respeta un orden cronológico lineal, sino que asume la espiralidad de la historia, en la cual podemos encontrar repeticiones de las mismas categorías como enunció Hegel y lo hemos sostenido en escritos previos. En efecto, a partir de una frase publicada en el periódico El Espectador aproximadamente en 1978, el artículo se devuelve a la conquista de Suramérica en donde la misma tensión emerge con otra cara, la de los traficantes de esclavos, luego salta a las propuestas teóricas y empíricas de un autor del siglo xix en Estados Unidos, considerado fundador de una escuela psicológica, para considerar después las propuestas originarias de la psicometría, que son traídas de Europa, desde la escuela ginebrina a nuestro país por Mercedes Rodrigo Bellido, quien es considerada fundadora de la primera facultad de psicología en el país. El artículo revisa entonces los propósitos e intenciones que pudo tener Mercedes Rodrigo y los contrasta con las ideas eugenésicas de algunos pensadores de aquella época y de hoy en día.

Palabras clave: historia, psicología, genealogía, tensión, emergencia. 
Mientras la historia del mundo siga su curso

lógico, dejará de cumplir su destino humano.

Max Horkheimer

Estado autoritario

\section{Introducción}

Emmanuel Kant sentenció, en una de las formulaciones del imperativo categórico, que debemos considerar a los demás siempre como un fin en sí mismos y nunca como un medio, también advirtió que debemos tratar a los demás como nosotros mismos deseamos ser tratados. La reciprocidad es el principio ético que estructura estas dos proposiciones.

Iniciaré con una anécdota: cuando entré a estudiar psicología en la Pontificia Universidad Javeriana en 1977, mi familia estaba suscrita al periódico El Espectador, el cual yo leía con fruición los domingos, en parte porque disfrutaba enormemente con las mordaces y certeras caricaturas de Héctor Osuna, en parte porque gozaba con una sección en la página editorial llamada "Microlingotes", en la que publicaban algunas frases cuyo hondo contenido dejaba pensativo al lector. De ese momento, si bien no tengo la fecha ni el número de la página, si recuerdo vívidamente una frase que quiero compartir con ustedes para dar inicio a esta conversación: "Yo estudio psicología para entenderme a mí mismo, y veterinaria para entender a los demás".

Aclaro que la frase era así, allí no decía: "y etología para entender a los demás”, lo cual podría haber matizado el significado de la denuncia que el periódico estaba haciendo con valor y sinceridad respecto de la actitud de algunos psicólogos, una actitud de pretendida superioridad, supremacía, desprecio, dominio, discriminación y exclusión. Por supuesto, en esta frase no se aplica ninguno de los mencionados principios formulados por Kant.
A la usanza genealógica de Nietzsche y de Foucault, les propongo develar algunas tensiones fundantes de nuestra disciplina, que renuevan las mismas cuestiones expresadas hasta aquí, de las cuales proceden y mediante las cuales emergen reiterativamente. Es por ello que quiero mencionar algunos momentos de nuestra historia como disciplina y como profesión, en los cuales se genera una tensión que coincide en sus categorías básicas con la frase citada y que son fruto de algo que no resulta evidente a primera vista y es que epistemología y política son sinónimos (Hoyos, 1980).

\section{Una manifestación de tal tensión en la Conquista y la Colonia en Suramérica}

No seguiré un orden cronológico sino genealógico para patentizar la espiralidad de la historia. El primer momento que quiero comentarles transcurre durante la conquista y la colonia de América y queda ilustrado en la acalorada discusión entre los traficantes de esclavos y las autoridades de estas épocas. A los traficantes les convenía que se aceptara oficialmente que los indígenas y los africanos no tenían alma, pues así podrían esclavizarlos y venderlos como animales sin la oposición de la ley, sin el juicio de la religión ni la censura de los ciudadanos. Permítanme un juego de palabras: los "desalmados" querían robar el alma a sus congéneres para hacerlos pasar por animales y así mejorar las utilidades de su macabro negocio. Paradójicamente, la etimología de la palabra "animal" nos revela precisamente que son criaturas con ánima.

Al respecto pueden encontrarse desgarradores testimonios en biografías como la de Pedro Claver S.I. (1580-1654), quien defendió a los negros traídos de África para ser esclavizados, sosteniendo que tenían alma y por ello mismo los bautizaba aun en contra de la voluntad de 
gente con poder, que prefería seguir considerándolos animales en defensa de sus propias prerrogativas tal y como se puede constatar en documentos históricos acerca de la evangelización en América Latina (Patiño, 2002).

Hoy en día, distintas legislaciones prohíben el tráfico de animales para preservar el equilibrio ecológico, pero en ese momento la exuberancia del paisaje permitía creer en la infinitud de los recursos naturales y era bien visto que se explotaran, incluyendo a los animales, y como ya vimos, a los seres humanos considerados como tales.

La etimología de la palabra "psicología" con p nos transporta a otros momentos de las historias de las psicologías en los cuales se presuponía la constitución múltiple y diversa de los seres humanos. Hoy podemos afirmar, atendiendo a nuestra complejidad, que cada ser humano es una unidad biopsicosocial indivisa. En la antigua Grecia se pensaba que al morir, el espíritu abandonaba el cuerpo por las heridas de los muertos en combate, a quienes dibujaban tirados en el piso y por sus laceraciones salían, volando libélulas, que fueron luego representadas gráficamente en la vigésimo tercera letra del alfabeto griego: $\Psi=$ psi. Ella nos recuerda al espíritu, al alma, al soplo de vida, al élan vital del profesor Henri Bergson, al hálito, al soplo, al aliento. En efecto, el verbo psiquein quiere decir soplar.

Espíritu viene del sustantivo latino spiritus (viento); y éste, del verbo spirare (soplar); que da origen a aspirar, expirar, así como a transpirar, conspirar, suspirar. Origen paralelo tiene en griego psiche (hálito, aliento) del cual se forma nuestro término psicología, que viene del verbo psychein (soplar, exhalar) (Barrera, 2005, p. 46).

¿Vamos a olvidar la bellísima tradición y a simplificar la escritura del nombre de nuestra disciplina quitándole la p inicial hasta borrar toda referencia al alma? ¿Solo algunos privilegiados gozamos del espíritu y los demás no? ¿Solo a algunos nos fue insuflado el soplo vital? Cuando la muerte nos llegue, ¿'solo a algunos cuerpos los dejará el espíritu? ¿Solo a algunos les saldrá volando por sus heridas el alma en forma de libélula, aquella que inspiró al calígrafo griego la vigésimo tercera letra de su antiguo alfabeto?

Toda la encíclica Laudato Si, Signore Mio, del Papa Francisco, está dedicada al respeto y el cuidado de la casa común, de la naturaleza, con la cual hemos mostrado tanta indolencia quizá por haber considerado precisamente que los seres humanos somos el centro de la creación y podríamos, incluso con respaldo en las Sagradas Escrituras, crecer y multiplicarnos y regodearnos "dominando" la tierra, precepto del "Génesis" que es discutido a profundidad por el papa Francisco desde una teología renovada.

Así pues, la de si los indígenas y africanos tenían o no alma, no fue una discusión meramente metafísica, un debate abstracto entre quienes defendían una postura dualista: alma-cuerpo, mente-soma, espíritu-materia, lo psicológico y lo fisiológico, y quienes defendían una postura monista materialista o espiritualista. Esta fue en realidad una discusión interesada, ambiciosa, enraizada en el barro de la lucha por el poder y por la riqueza, que buscaba resolver en el plano ontológico asuntos económicos, jurídicos y políticos de predominio y dominación de unos seres humanos sobre otros.

Fijémonos en que en estos acontecimientos, se revela una tensión genealógica, la misma que quedó plasmada en la frase aludida de $E l$ Espectador, una tensión que se manifiesta también en otros momentos de la historia, de los cuales puede inferirse que cuando los psicólogos entramos en discusiones ontológicas acerca de qué es lo psicológico y qué estudia la psicología. En discusiones epistemológicas sobre cómo se puede conocer lo psicológico o en

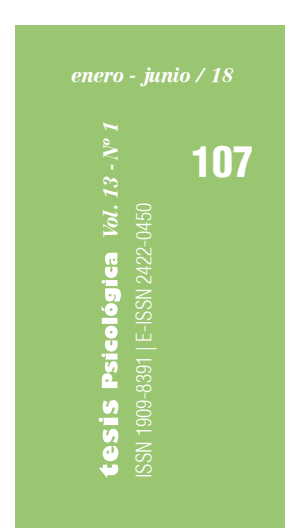


discusiones acerca del método para estudiar lo psicológico, conscientes o no, deliberadamente o no, nos atraviesan cuestiones antropológicas — ¿cómo concebimos a los seres humanos?

—, cuestiones éticas — ¿qué es bueno y qué no? - y cuestiones políticas — ¿para quién es útil el conocimiento producido?-, cuestiones para cuya develación será necesario identificar las coordenadas, el contexto, las condiciones, los intereses y las tensiones en que se producen y se generan los conocimientos psicológicos.

Será necesario pues conocer las trayectorias, las emergencias y las procedencias de las fuerzas que nos impelen a tomar opciones, aquellas que inclinan a un psicólogo por un paradigma o por otro, que lo llevan a tomar decisiones acerca de aquello que considera que es lo psicológico y por la manera válida y legítima de estudiarlo.

\section{El relato fundacional de la psicología en Colombia}

Voy a describir otros acontecimientos ocurridos en la historia de las psicologías en Colombia, en los cuales se manifiesta la misma tensión ya descrita someramente en dos épocas muy distantes en el tiempo, para lo cual les invito a considerar un momento estimado como primordial para la psicología como disciplina y como profesión en nuestro país.

El relato fundacional de la psicología en Colombia incluye la narración básica de la invitación de la psicóloga española Mercedes Rodrigo Bellido al país. Ella llegó a fundar y dirigir la Sección de Psicotécnia que luego sería el Instituto de Psicología Aplicada de la Universidad Nacional. En la Sección de Psicotecnia se diseñaron y aplicaron las primeras pruebas para realizar la selección de los aspirantes a estudiantes en las facultades de Medicina, Derecho e Ingeniería de dicha universidad.
Recordemos que en el ambiente de la época y desde el siglo XIX ${ }^{1}$ algunos autores querían que la psicología, para que fuera considerada ciencia, se asemejara a las ciencias naturales (Valenzuela, 1980). Autores considerados fundadores de la psicología científica como Fechner, Wundt, Pavlov y Titchener (Gondra, 1990) entre otros, buscaban que la psicología calcase a la biología, la fisiología o la física para ser reconocida como ciencia. En este sentido, afirmaciones como la que hizo James McKeen Catell en 1890 trazaban una ruta para la psicología que quisiese llamarse científica y en particular para la psicometría, según los cánones de la época:

La psicología no puede alcanzar la certeza y exactitud de las ciencias físicas si no se funda en el experimento y en la medida. Se podría dar un paso en esta dirección aplicando una serie de test y medidas mentales a un gran número de individuos. Los resultados serían de gran valor científico al descubrir la constancia e interdependencia de los procesos mentales, y sus cambios en las distintas circunstancias (Catell, 1890, p. 373 citado por Tortosa \& Civera, 2006, p. 172).

Si bien la psicometría es una opción por un modelo de caja traslúcida, en el cual se postulan procesos y entidades internas - constructossusceptibles de conocerse por medio de los test y de la hermenéutica, en vista de que no pueden ser observados directamente. Esto hace que históricamente la psicometría esté más relacionada con el racionalismo e incluso con el idealismo - en ese párrafo de Catell se pone en evidencia - , dado que propone que la psicología imite a las ciencias naturales y copie su pretensión de exactitud, una opción epistemológica naturalista, positivista. Una opción por una forma de concebir a los seres humanos y de hacer ciencia, de hacer psicología, un paradigma empíricoanalítico con preferencias por lo cuantitativo,

1 Mercedes llega al país en agosto de 1939, a la Colombia que en tan solo nueve años estallaría en el bogotazo y se va del país en 1950 . 
cuyo interés quedó definido explícitamente años después en la obra de autores como John Broadus Watson (1913, en Gondra, 1990), cuando definió a la psicología como "una rama experimental puramente objetiva de las ciencias naturales", cuya finalidad era estudiar la conducta observable con el propósito de predecirla, controlarla y modificarla.

El modelo de caja negra traza sus límites y sus métodos cuando define que lo que estudia la psicología es la conducta observable y que lo hace a través de la experimentación. Si bien no todos los conductismos toman decisiones así de radicales y pueden esgrimir otros propósitos y métodos diferentes, para evidenciar mi punto de vista resalto aquí la tensión que quiero presentarles: unos seres humanos se atribuyen el poder de predecir, controlar y modificar la conducta de otros seres humanos: "yo estudio psicología para entenderme a mí mismo, y veterinaria para entender a los demás”.

Así pueden constatarse siguiendo tensiones genealógicas, diferentes vertientes en las historias de las psicologías. Bruno Andrés Jaraba Barrios, en su agudo estudio acerca del mito fundacional de la psicología en Colombia, señala críticamente respecto de las primeras pruebas psicométricas aplicadas en el país:

\footnotetext{
A partir de lo encontrado, se puede afirmar que, al contrario de lo sostenido por el relato histórico vigente, los exámenes de admisión fueron menos decisivos para la constitución de la comunidad psicológica nacional que para la definición de la política limitacionista que hasta el día de hoy caracteriza al sistema universitario público en Colombia (Jaraba, 2014, p. XI).
}

En otras palabras, los criterios psicométricos fueron utilizados para justificar decisiones excluyentes y políticas limitacionistas del ingreso a la universidad, disfrazándolas con un tratamiento y un lenguaje "científicos". Jaraba señala que en lugar de considerar ampliar las instalaciones o aumentar el número de profesores, se decidió recortar el número de estudiantes admitidos y para ello nada mejor que la selección por medio de pruebas psicométricas. Aunque siempre podrán justificarse tales decisiones con argumentos económicos y administrativos, podemos preguntarnos: ¿Mercedes Rodrigo estaba cómoda con estas decisiones y estas prácticas? ¿Qué caracterizaba el contexto histórico colombiano cuando Mercedes Rodrigo funda el Instituto de Psicología Aplicada?

Algunos historiadores señalan que en Colombia desde 1920 tuvo lugar una fuerte polémica acerca de la "degeneración" de la raza colombiana (Peña, 1993). Factores como el darwinismo social resultaban ser un poderoso aliciente a las decisiones discriminatorias con el pretexto de garantizar "la salud" de la población. Pueden verse al respecto algunos escritos de Luís López de Mesa, quien animó tal polémica desde los cargos directivos que ocupó en diversos gobiernos, en los cuales tomó decisiones coherentes con sus ideas. En esos escritos se instaura una postura a favor de la eugenesia, que para este autor consistía en que los colombianos se mezclaran con algunos pueblos europeos para mejorar la raza, pero no con los judíos.

En tal polémica hubo posturas a favor de la eugenesia, de personas interesadas en seleccionar y preservar aquello que consideraban "lo mejor" de la raza y de excluir e incluso eliminar aquellas características que consideraron "degeneradas". Aunque también hubo posturas afines con estudios previos como los del sabio Francisco José de Caldas, que defendieron la calidad de las razas nativas y las posibilidades de su hibridación. Notemos que esta es una nueva manifestación, una nueva mostración, una actualización, esta vez vestida de cientificidad, de la misma tensión genealógica que veníamos mencionando. 
¿En ese contexto, qué pretendían Mercedes Rodrigo y sus colegas haciendo medición y evaluación? Para responder a esta compleja pregunta es preciso examinar algunos orígenes de la psicometría en América:

\footnotetext{
El test de Binet llegó a América de la mano de otro eugenista, Goddard, director del Laboratorio del Centro de Formación para Débiles Mentales en Vineland (New Jersey) (...) Buena prueba de su éxito fue que, en 1910, (...) la Asociación Americana para el Estudio de la Deficiencia Mental adoptó los test de inteligencia como su principal criterio para el diagnóstico de la debilidad mental (Zenderland, 1987, p. 46 citado por Tortosa \& Civera, 2006, pp. 172 y 173).
}

No solo se utilizaron las pruebas recientemente construidas para realizar las evaluaciones diferenciales de debilidad mental, sino que sus resultados fueron utilizados en Estados Unidos como criterio para esterilizar a aquellas personas consideradas débiles mentales a fin de que no pudieran reproducirse; además, fueron criterios para decidir políticas de inmigración con fines discriminatorios de exclusión (Tortosa \& Civera, 2006).

\section{Intelligence Classification of Immigrants of Different Nationalities}

Estos mismos autores citan más adelante a Samelson, quien en 1975 señaló: “iii Ya comentamos el mal uso, totalmente contrario a lo que su autor deseaba, de la Escala de Binet en las manos (instrumentalmente politizadas) de los Eugenistas!!!” (Tortosa \& Civera, 2006, pp. 172 y 173).

Son estos mismos historiadores de la psicología quienes señalan que las políticas de inmigración, altamente discriminatorias, encontraron un pretexto en el uso del test de Binet, uso cuestionable tanto por su tergiversación de los propósitos originales de la prueba, como por sus intenciones políticas excluyentes con ropaje científico. Hay indicios de que también en Colombia la eugenesia fue criterio para definir políticas de inmigración y de ingreso a la educación.

¿Revivimos esta tensión en la actualidad con los acontecimientos más recientes que vemos en Estados Unidos respecto de sus fronteras y sus políticas internacionales, en particular las de inmigración?

\section{¿Puede la psicología pretender ser neutra o desinteresada ética o políticamente?}

Autores como Jaraba (2104) y Herrero (2003) dejan ver que en la Colombia en que vivió Mercedes Rodrigo, algunos defensores de la eugenesia tenían gran influencia en las decisiones de política internacional, pública, educativa y de educación universitaria. ¿¡Fue Mercedes Rodrigo, en calidad de trabajadora subordinada, libre para tomar decisiones a favor de la democratización y en contra de la eugenesia? ¿Cómo describir la vivencia de esa tensión que debió caracterizar el ejercicio profesional de Mercedes Rodrigo? Sale de España exiliada por sus ideas democráticas contrarias a las de la dictadura del Franquismo, la invita a Colombia un pensador amigo de las reformas educativas de la Escuela Nueva pero también de las decisiones limitacionistas en cuestiones de ingreso a la universidad trabaja como subordinada a un grupo de personajes influyentes en la medicina y en la educación que ganaron fama de eugenistas. ¿Por qué sale de Colombia aproximadamente diez años después de su llegada y en qué circunstancias y condiciones? ¿Qué sentimientos atravesaron el pecho de Mercedes Rodrigo? ¿De qué maneras resolvió los asuntos fundamentales para la psicología que recién nacía como disciplina y como profesión en Colombia? ¿Qué asuntos ontológicos, epistemológicos, metodológicos, 
éticos y políticos albergaban sus preguntas de "psicología aplicada"?

La especialidad de Mercedes Rodrigo fue la "orientación vocacional" más que la "selección profesional", interés que heredó de Claparéde, de Piaget y de otros maestros de la Escuela Ginebrina y que ella trajo a nuestro país. Ella quería que la orientación vocacional se aplicara desde la escuela primaria, pues estaba convencida de que el desarrollo y la felicidad del individuo se alcanzarían articulando los propósitos individuales con las demandas del sector económico, conciliando el interés colectivo y el individual. Pero hacía énfasis en la prevalencia del interés individual sobre el colectivo, dentro de una concepción de tipo humanista-socialista que buscaba armonizar el deseo del estudiante y sus aptitudes con el deseo de sus padres y los de la sociedad (Herrero, 2003).

De este modo, para ella la sociedad debería ser posibilitadora del desarrollo personal y la orientación vocacional debería tener el carácter de derecho ciudadano fundamental desde la infancia, pues resulta trascendental para la vida personal del individuo, para la realización de su proyecto de vida. Estas características de su pensamiento indican que Rodrigo tomó distancia crítica del uso de la psicometría como estrategia eugenésica o limitacionista (Herrero, 2003).

Su opción por democratizar la sociedad le hizo pensar en no limitar las elecciones personales desde temprano y proponer el incluir todas las carreras y profesiones. Para ella la orientación vocacional es al mismo tiempo un asunto psicofisiológico, individual, familiar y social para que un individuo adolescente escoja profesión. La selección profesional, por su parte, es escoger a un individuo adulto capaz para ejercer una profesión. Ambas son un pronóstico que el tiempo se encargará de confirmar o desmentir (Herrero, 2003, p. 162). Pronosticar esperando la posibilidad de equivocarse y respetando la libre voluntad de las personas es algo muy lejano del predecir, controlar y modificar. Su idea de incidir en el curso de la historia era contraria a una concepción lineal, unidireccional, pues buscaba una sociedad democrática e igualitaria, contraria a una idea piramidal de la sociedad:

\footnotetext{
Todavía se hacen aprender de memoria listas de reyes y series de guerras (...) falsos patriotismos y absurdos nacionalismos que nos llevaron a la hecatombe de 1914 (...) en vez de buscar, precisamente por la historia del trabajo, la unión y la comprensión de todos los hombres de la humanidad (Rodrigo, 1934, citada por Herrero, 2003, p. 186).
}

Respecto de la linealidad de la historia de la psicología y lo que así se nos muestra y lo que así se nos oculta, Camilo Pulido (2011) devela con elocuencia:

\footnotetext{
esta forma lineal de considerar la historia de la disciplina sirve básicamente para vigilar sus límites. Es decir, las historias celebrantes y oficiales cumplen el papel de establecer un cierto control sobre el porvenir de la disciplina, al determinar temas y métodos del pasado que son valiosos de conservar hacia el futuro (p. 64).
}

\section{Espiralidad de las historias}

Parece pues que la historia no avanza como una flecha en un solo sentido, sino que la espiralidad hegeliana de sus procesos permite encontrar varias mostraciones de la misma tensión, la cual brota a través de diferentes manifestaciones en distintos momentos. Es notorio el énfasis de Rodrigo en la autodeterminación y en la autogestión que permiten al sujeto dar dirección a la biografía y a la historia, superando una perspectiva determinista.

Rodrigo rompía la linealidad que supone asumir que somos espectadores pasivos de un

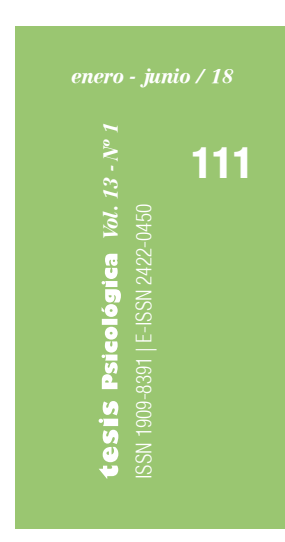


transcurrir monótono de la historia en el cual otros son los protagonistas, preguntando a los niños qué querían ser cuando grandes, invitándolos a la futurición (Bertrand Russell), favoreciendo en ellos la implementación de un proyecto de vida realista y a la vez sońador, construyendo con ellos la voluntad, órgano de la libertad. No hay más que revisar el Cuestionario de los ideales (Herrero, 2003, pp. 166 y 167) con las preguntas que hacía a los niños, con el cual centraba su atención en la persona, sus decisiones y su desarrollo. Por eso recomendaba ampliar la orientación vocacional en tiempos de democratización sin olvidar las imposiciones económicas en épocas de crisis (Herrero, 2003, p. 182).

Quizá Louis Not (1992) podría decir que Rodrigo favorecía en los niños el desarrollo de la "conducta de proyecto". También podemos afirmar que Mercedes Rodrigo procuraba la constitución de las personas en sujetos de su proyecto histórico concreto, a la usanza de la Escuela Ginebrina y de pensadores anteriores como Pestalozzi o defensores del liberalismo como Russell o educadores como Montessori, Decroly y Freinet, cuya obra luego resonó en el pedagogo brasilero de la liberación Paulo Freire, potenciando la visualización de la misma persona a corto, mediano y largo plazo, favoreciendo así la construcción de planes, proyectos y del sentido vital. Podríamos afirmar que Rodrigo estaría de acuerdo hoy con la Orientación Temporal de la Personalidad.

Quizá sus intenciones de respetar la autodeterminación del individuo, la búsqueda de una sociedad universalista y anticlasista, democrática de estilo ginebrino y su afán de participar en una organización racional del trabajo le significaron tensiones y presiones de algunos defensores de la eugenesia debido a las cuales decide emigrar a Puerto Rico, donde finalmente muere. ¿Con cuáles intereses económicos y políticos se confrontó en Colombia? ¿Qué rigideces o flexibilidades encontró en su camino?

Frente a la llamada "deficiencia mental", Rodrigo propuso una intervención educativa contraria a la eugenesia. Voy a tomar un ejemplo de la tesis doctoral de Fania Herrero (2003) para ilustrar su valiente y arrojada posición. En respuesta a un ponente en una conferencia, quien advierte que los deficientes mentales pueden producir un daño incalculable sin intención, Mercedes responde:

Por mi parte..., pensando que los deficientes mentales son incapaces de defenderse ellos mismos y de organizar ninguna acción en defensa de su propia causa,... parece de justicia ocuparse de ellos puesto que .... (Usted) Quiere decir que los desventurados oligofrénicos pueden causar 'daño incalculable' si no cumplimos con nuestro elemental deber cristiano de amar al prójimo como a nosotros mismos (Rodrigo, 1954, p. 109, en Herrero 2003, p. 383).

En Puerto Rico, refiriéndose al C.I. como la única medida que se consideraba allí para hacer el diagnóstico y la posterior intervención con niños con dificultades, Mercedes indica:

Frente a las cifras clasificatorias de un escolar, atendiendo al resultado de estas pruebas, que "francamente suenan como a marcas en el ganado", Mercedes Rodrigo afirma que "un niño no puede ser un número" y menos cuando tal número puede variar ante una gran cantidad de factores de salud, emotivos incluso en sus relaciones con el examinador, el grado de fatiga, la situación familiar, etc. (Rodrigo, 1954, p. 109 en Herrero, 2003, p. 384) 2 .

¿Cómo buscó Mercedes superar las evidentes contradicciones entre su intención de contribuir con la democratización a través de su

2 Negrilla del autor para subrayar la tensión genealógica que vuelve a aparecer insidiosamente en la historia de las psicologías. 
esquema de orientación y las pretensiones eugenésicas de la política en Colombia o en otras latitudes? ¿Por qué y cómo fueron trasladados los test desarrollados por Rodrigo y su equipo desde la universidad, hacia el mundo gubernamental, educativo e industrial con tanta rapidez? ¿Qué intereses caracterizaron las decisiones del Instituto de Psicología Aplicada?

La Escuela de Frankfurt nos permite pensar en que tras de la psicometría puede haber afanes de conocimiento, interpretación, comprensión y ubicación para la praxis social (interés práctico); intenciones de liberación y emancipación de las ataduras de la opresión (interés emancipatorio), pero también puede haberlos de predicción, manipulación y control (interés técnico).

¿Qué intensiones teóricas (epistemológicaséticas-políticas) se escondían detrás de una perspectiva "aplicada" de la psicología? ¿Cómo articuló Rodrigo sus ideas humanistas con la concepción cuantitativa de una psicología con pretensiones de ser aplicada? ¿Quedaron velados los intereses políticos detrás de los intereses científicos? ¿Se frustró la contribución de Mercedes Rodrigo a la organización social, debido a la intervención de personas con posturas dogmáticas, que vieron amenazados sus intereses personales y colectivos por la argumentación democrática de Rodrigo? ¿Quiénes consideraron amenazantes las sólidas argumentaciones y las fundadas propuestas de Mercedes? ¿Había/ hay en Colombia personas que se mantienen en el poder gracias a la psicología, mientras consideran que para gobernar a los demás es necesario aplicar la veterinaria?

\section{Conclusión}

Una frase en un periódico colombiano en 1978, traficantes de esclavos durante la Conquista, un investigador norteamericano de inicios del siglo $\mathrm{xx}$, un antiguo calígrafo griego, la Sección de Psicotecnia de la Universidad Nacional, la utilización de la psicometría por parte de políticos nacionales y extranjeros, ¿qué tienen en común? Que todos reeditan, con caras diversas, la tensión antropológica fundamental que define nuestras opciones en psicología. Notemos que todas las preguntas que dan origen a nuestra disciplina son políticas y entonces no es legítimo postular una pretendida neutralidad.

Sé que les dejo más preguntas que respuestas: ¿cómo recoger las propuestas de Mercedes Rodrigo en la Colombia del postconflicto? ¿Cómo puede la psicología hoy responder con sistematicidad y rigor desde la reflexión crítica y a la vez con esperanza en el futuro, a la tensión entre la diversidad y la unidad que caracteriza la dejación de las armas? ¿Debe responder (es decir ser responsable) una universidad pontificia, como la Bolivariana o la Javeriana en la tónica de la encíclica Laudato Si, a la complejidad biopsicosocial que nos constituye en la unidad con orientación vocacional más que con selección? ¿Cuál es la mejor manera para hacer inclusión en conformidad con nuestro amor cristiano?

Una posible conclusión de esta charla: dijo Henri Bergson en 1960 que no pueden extenderse arbitrariamente a las acciones voluntarias, leyes que han sido comprobadas en casos en los cuales la voluntad no interviene.

Todo determinismo en psicología es reduccionista y todo reduccionismo desconoce la complejidad de los seres humanos y su libertad decisoria. Debemos poner en psicología, entre nuestros principales propósitos, al estudio psicológico de la libertad. Solo asumiendo este programa podremos dar respuestas digna y competentemente a tan acuciantes preguntas.

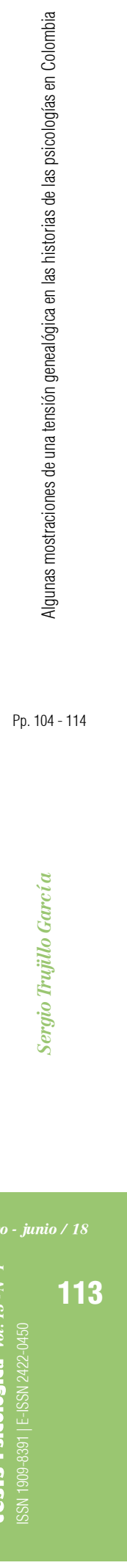




\section{Referencias}

Barrera, J. (2005). El cuidado del espíritu. En La educación desde las éticas del cuidado y la compasión (pp. 45-52). Bogotá: Editorial Universidad Javeriana.

Gondra, J.M. (1990). La psicología moderna. Textos básicos para su génesis y desarrollo histórico. Bilbao: Desclée de Brouwer.

Herrero, F. (2003). Mercedes Rodrigo: una pionera de la psicología aplicada en España y Colombia (tesis, Universidad Complutense de Madrid, Madrid, España).

Jaraba Barrios, B.A. (2014). Un escritorio para Mercedes: revisando el mito fundacional de la psicología en Colombia (tesis de maestría, Universidad Nacional de Colombia, Bogotá, Colombia).

Not, L. (1992). La enseñanza dialogante. Hacia una educación en segunda persona. Barcelona: Herder.

Patiño, J. U. (2002). La Iglesia en América Latina: una mirada histórica al proceso evangelizador eclesial en el continente de la esperanza, Siglos XV-XX. Bogotá: San Pablo.

Peña, T. (1993). La psicología en Colombia: Historia de una disciplina y una profesión. En Historia Social de la Ciencia en Colombia. Instituto Colombiano para el desarrollo de la Ciencia y la Tecnología Francisco José de Caldas, Colciencias, Tomo IX.

Pulido, H. C. (2011). Una reflexión sobre la relación psicología - trabajo desde una perspectiva histórica. En S. Trujillo \& L. M. Carvajal. Historias y debates de las psicologias en Colombia (pp. 63-76). Bogotá: Editorial Pontificia Universidad Javeriana.

Tortosa, F. \& Civera, C. (2006). Historia de la psicología. Madrid: McGraw-Hill. 